\title{
Sutureless aortic valve prosthesis in a calcified homograft
}

\author{
Filippo Capestro, MD, ${ }^{a}$ Simone Massaccesi, MD,${ }^{b}$ M. L. Sacha Matteucci, MD, ${ }^{a}$ and Lucia Torracca, MD, \\ Ancona, Italy
}

See related commentary on page e31.

Surgical aortic valve replacement (AVR) is the treatment of choice for severe aortic valve regurgitation. In patients undergoing reoperation with a severe calcified homograft AVR is a challenging operation, and alternative solutions are prompted for the high surgical risk. Predominant aortic valve regurgitation remains a relative contraindication for transcatheter aortic valve implantation. The use of sutureless aortic valves offers a viable option for these patients at high risk with conventional approaches. The potential clinical advantages are related to the easier implantation, specifically in completely calcified aortic roots, with reduced extracorporeal circulation and aortic crossclamp times. We report a case of sutureless aortic valve implantation within a calcified aortic root homograft.

\section{CLINICAL SUMMARY}

The patient was a 71-year-old man with severe aortic regurgitation as a result of homograft degeneration. He was hospitalized in our cardiology department for congestive heart failure. Transthoracic echocardiography showed severe aortic valve regurgitation, left ventricular dilatation with an end-diastolic volume of $121 \mathrm{~mL} / \mathrm{m}^{2}$, mildly depressed left ventricular function with an ejection fraction of 0.46 , and a calcified aortic root.

He had been operated on 19 years previously for native aortic valve endocarditis as a result of Staphylococcus aureus infection and received an aortic homograft. His comorbidities included hypertension, diabetes, previous right nephrectomy (glomerular filtration rate $>50 \mathrm{~mL} /$ $\mathrm{min}$ ), and previous partial gastrectomy. His calculated logistic euroSCORE was $23.9 \%$, and his Society of

\footnotetext{
From the a Division of Cardiac Surgery, Azienda Ospedaliero-Universitaria Ospedali Riuniti, Ancona, Italy; and the ${ }^{\mathrm{b}}$ Division of Postoperative Intensive Care, Azienda Ospedaliero-Universitaria Ospedali Riuniti, Ancona, Italy.

Disclosures: Authors have nothing to disclose with regard to commercial support.

Received for publication Feb 15, 2015; revisions received April 1, 2015; accepted for publication April 12, 2015; available ahead of print May 9, 2015

Address for reprints: Filippo Capestro, MD, Department of Cardiac Surgery,

Azienda Ospedaliero-Universitaria, Via Conca 71-60020, Ancona, Italy

(E-mail: filippocapestro@libero.it).

J Thorac Cardiovasc Surg 2015;150:e29-30

$0022-5223 / \$ 36.00$

Copyright (c) 2015 by The American Association for Thoracic Surgery

http://dx.doi.org/10.1016/j.jtcvs.2015.04.025
}

Thoracic Surgeons calculated risk of mortality was $2.4 \%$. Coronary angiography showed no significant stenosis of epicardial branches. A computed tomographic scan was performed to evaluate the ascending aorta (Figure 1); it revealed a severe calcified aortic root. The calcification began $5.5 \mathrm{~cm}$ above the plane of the aortic valve. He was admitted for surgical AVR. We decided to implant a stentless sutureless valve aortic prosthesis because the aortic annulus presented massive calcifications and the use of standard suture stitches, required for a conventional prosthesis, could have been troublesome in this clinical scenario.

After induction of general anesthesia, the right femoral vessels were isolated as a precautionary measure. A median sternotomy was performed and, after pericardial adhesions lysis, the patient was placed on cardiopulmonary bypass through cannulation of the proximal aortic arch and right atrial appendage. A left ventricular vent was inserted in the superior right pulmonary vein. The aorta was clamped, and cold crystalloid cardioplegia was administered, first in a retrograde fashion and then, after aortotomy, directly into the coronary ostia. The aorta was opened $1.5 \mathrm{~cm}$ above the homograft suture line. A massively calcified aortic root and a torn right coronary leaflet were documented. Because of the heavy calcification of the aortic annulus around the coronary ostia and the proximal part of the ascending aorta a traditional AVR procedure was considered very challenging and dangerous. The aortic leaflets were excised and, after sizing, a Perceval S valve (Size L; Sorin Biomedica Cardio Srl, Sallugia, Italy) was implanted.

The aortotomy was closed with a running suture line, and the operation was terminated in the usual fashion. The crossclamp time was 51 minutes. Position and function of the valve were assessed by intraoperative transesophageal echocardiography immediately after weaning from cardiopulmonary bypass, which showed no perivalvular leakage and a good prosthetic valve function (Figure 2). The postoperative course was complicated by reexploration for bleeding as a result of thoracic vein lesion during sternal closure. The patient was discharged on the 8th postoperative day.

\section{DISCUSSION}

The current treatment choice for severe aortic valve stenosis remains AVR with valvular prostheses, which are hand sewn to the aortic annulus with a series of stitches. The sutureless aortic valve bioprosthesis have been 


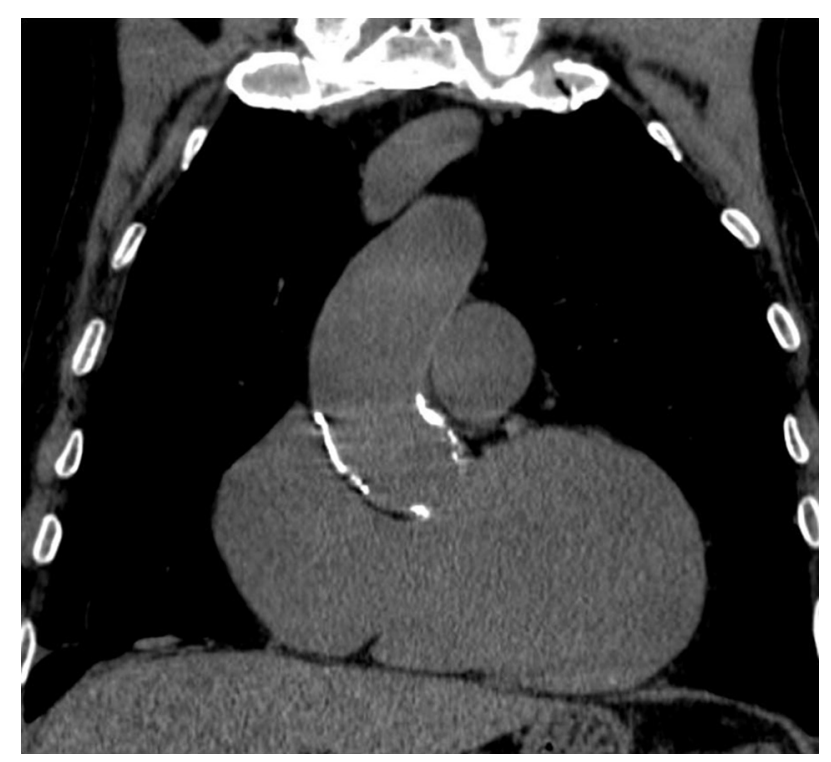

FIGURE 1. Computed tomographic scan shows a severely calcified aortic root.

designed to allow simple and fast surgical implantation; however, the role of these bioprostheses in cardiac surgery is still a matter of debate. Sutureless aortic valves are gaining interest as an important option for AVR in high-risk patients. The fast implantation of the device reduces the crossclamp time and the cardiopulmonary bypass time, and in high-risk patients this could give a better outcome. ${ }^{1,2}$

Moreover, these devices offer the potential for simplifying less invasive approaches. With the advantages of minimally invasive access AVR, these devices thus could evolve into the standard surgical technique for $\mathrm{AVR}^{3}$ Furthermore, the use of a sutureless valve in selected cases, such as the case reported by us, can be considered necessary. In fact, the massive calcification of the annulus in such cases makes the suturing of a conventional prosthesis extremely demanding and poses a high

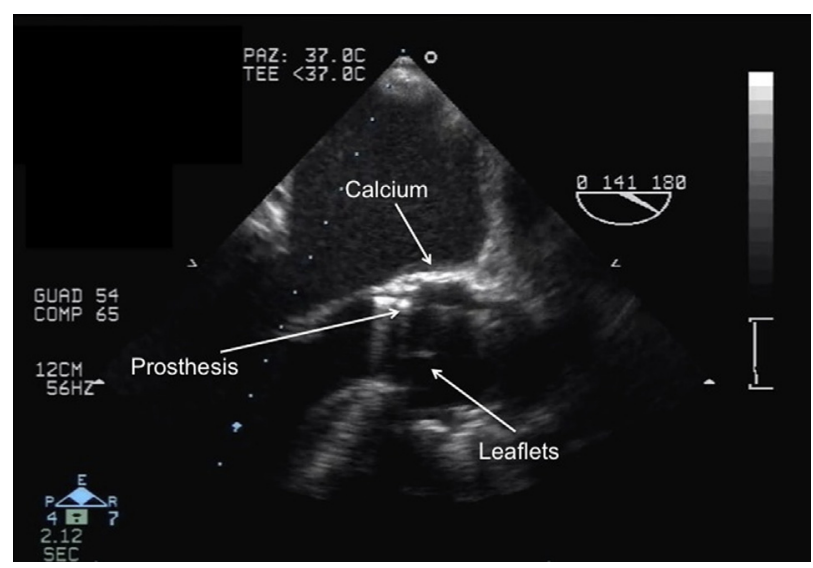

FIGURE 2. Postoperative transesophageal echocardiography.

risk of intraoperative and postoperative complications. In addition, performing the aortotomy very far from the annulus because of massive root calcification makes the exposure very poor. The surgical approach, as described here and by others, ${ }^{4,5}$ is a technically simple alternative to conventional AVR and demonstrates feasibility in patients with severe calcification of the aortic root.

\section{References}

1. Rubino AS, Santarpino G, De Praetere H, Kasama K, Dalén M, Sartipy U, et al. Early and intermediate outcome after aortic valve replacement with a sutureless bioprosthesis: results of a multicenter study. J Thorac Cardiovasc Surg. 2014; 148:865-71.

2. Pollari F, Santarpino G, Dell'Aquila AM, Gazdag L, Alnahas H, Vogt F, et al. Better short-term outcome by using sutureless valves: a propensity-matched score analysis. Ann Thorac Surg. 2014;98:611-6; discussion 616-7.

3. Gilmanov D, Miceli A, Bevilacqua S, Farneti P, Solinas M, Ferrarini M, et al. Sutureless implantation of the Perceval S aortic valve prosthesis through right anterior minithoracotomy. Ann Thorac Surg. 2013;96:2101-8.

4. Concistrè G, Farneti P, Miceli A, Glauber M. Sutureless aortic bioprosthesis in severe aortic root calcification: an innovative approach. Interact Cardiovasc Thorac Surg. 2012;14:670-2.

5. Gupta P, McCormack DJ, Szczeklik M, Ambekar S, Lall KS. Infected calcified homograft root: a sutureless solution. Ann Thorac Surg. 2013;95: $1789-91$. 